\title{
SOME CHARACTERIZATIONS OF REAL HYPERSURFACES OF TYPE (A) IN A NONFLAT COMPLEX SPACE FORM
}

\author{
U-HANG KI AND HUILI LIU
}

\begin{abstract}
In this paper, we prove that if the structure Jacobi operator $R_{\xi}$ is $\xi$-parallel and $R_{\xi}$ commutes with the Ricci tensor $S$, then a real hypersurface with non-negative scalar curvature of a nonflat complex space form $\mathbf{M}_{n}(c)$ is a Hopf hypersurface. Further, we characterize such Hopf hypersurface in $\mathbf{M}_{n}(c)$.
\end{abstract}

\section{Introduction}

A complex $n$-dimensional Kähler manifold of constant holomorphic sectional curvature $c$ is called a complex space form, which is denoted by $\mathbf{M}_{n}(c)$. As is well-known, a connected complete and simply connected complex space form is complex analytically isometric to a complex projective space $\mathbf{P}_{n} \mathbb{C}$, a complex Euclidean space $\mathbb{C}_{n}$ or a complex hyperbolic space $\mathbf{H}_{n} \mathbb{C}$ according as $c>0$, $c=0$ or $c<0$.

In this paper, we consider a real hypersurface $\mathbf{M}$ in a complex space form $\mathbf{M}_{n}(c), c \neq 0$. Then $\mathbf{M}$ has an almost contact metric structure $(\phi, \xi, \eta, g)$ induced from the Kähler metric and complex structure $J$ on $\mathbf{M}_{n}(c)$. The structure vector field $\xi$ is said to be principal if $A \xi=\alpha \xi$ is satisfied, where $A$ is the shape operator of $\mathbf{M}$ and $\alpha=\eta(A \xi)$. In this case, it is known that $\alpha$ is locally constant [6] and $\mathbf{M}$ is called Hopf hypersurface.

Typical examples of Hopf hypersurfaces in $\mathbf{P}_{n} \mathbb{C}$ are homogeneous ones, namely those real hypersurfaces are given as orbits under subgroup of the projective unitary group $\mathbf{P U}(n+1)$. Takagi [13] completely classified such hypersurfaces as six model spaces which are said to be $A_{1}, A_{2}, B, C, D$ and E.

On the other hand, real hypersurfaces in $\mathbf{H}_{n} \mathbb{C}$ have been investigated by Berndt [1], Montiel and Romero [8] and so on. Berndt [1] classified Hopf

Received July 4, 2006.

2000 Mathematics Subject Classification. 53C40, 53C15.

Key words and phrases. real hypersurface, structure Jacobi operator, Ricci tensor, Hopf hypersurface.

The second author was supported by NSFC, No.10371013; Joint Research of NSFC and KOSEF, No. 10510637; Northeastern University. 
hypersurfaces in a complex hyperbolic space whose all principal curvatures are constant as four model spaces which are said to be $A_{0}, A_{1}, A_{2}$ and $B$.

We introduce the following theorem without proof which due to Okumura [10] for $c>0$, Montiel and Romero [8] for $c<0$ respectively.

Theorem O-MR. ([10], [8]). Let $\mathbf{M}$ be a real hypersurface of $\mathbf{M}_{n}(c), c \neq 0$. If it satisfies $A \phi-\phi A=0$, then $\mathbf{M}$ is locally congruent to one of the following hypersurfaces:

(1) In case $\mathbf{P}_{n} \mathbb{C}$

$\left(A_{1}\right)$ a geodesic hypersphere of radius $r$, where $0<r<\pi / 2$;

$\left(A_{2}\right)$ a tube of radius $r$ over a totally geodesic $\mathbf{P}_{k} \mathbb{C}(1 \leq k \leq n-2)$, where $0<r<\pi / 2$.

(2) In case $\mathbf{H}_{n} \mathbb{C}$

$\left(A_{0}\right)$ a horosphere in $\mathbf{H}_{n} \mathbb{C}$, i.e., a Montiel tube;

$\left(A_{1}\right)$ a geodesic hypersphere or a tube over a complex hyperbolic hyperplane $\mathbf{H}_{n-1} \mathbb{C}$;

$\left(A_{2}\right)$ a tube of a totally geodesic $\mathbf{H}_{k} \mathbb{C}(1 \leq k \leq n-2)$.

Let $\mathbf{M}$ be a real hypersurface of type $\left(A_{1}\right)$ or $\left(A_{2}\right)$ in a $\mathbf{P}_{n} \mathbb{C}$, or type $\left(A_{0}\right)$, $\left(A_{1}\right)$ or $\left(A_{2}\right)$ in a $\mathbf{H}_{n} \mathbb{C}$. Then $\mathbf{M}$ is said to be type $(A)$ for simplicity.

The curvature tensor field $R$ on a Riemannian manifold $(\mathbf{M}, g)$ is defined by

$$
R(X, Y)=\left[\nabla_{X}, \nabla_{Y}\right]-\nabla_{[X, Y]}
$$

for any vector fields $X$ and $Y$ on $(\mathbf{M}, g)$. We define the Jacobi operator field $R_{X}=R(\cdot, X) X$ with respect to a unit vector field $X$. Then we see that $R_{X}$ is a self-adjoint endomorphism of the tangent space. It is related with (the Jacobi vector equation) $\nabla_{\dot{\gamma}}\left(\nabla_{\dot{\gamma}} Y\right)+R(Y, \dot{\gamma}) \dot{\gamma}=0$ along a geodesic $\gamma$, where $\dot{\gamma}$ denotes the velocity vector field along $\gamma$.

When we study a real hypersurface $\mathbf{M}$ in a complex space form, we will call the Jacobi operator on $\mathbf{M}$ with respect to the structure vector field $\xi$ a structure Jacobi operator on $\mathbf{M}$ and will denote it by $R_{\xi}$, where $R_{\xi}$ is defined by $R_{\xi}(X)=R(X, \xi) \xi$. However, recently it is known that there are no real hypersurfaces in $\mathbf{M}_{n}(c)$ with parallel Jacobi operator $R_{\xi}$ (see [11] and [12]). Some works have recently studied several conditions on the structure Jacobi operator $R_{\xi}$ and given some results on the classification of real hypersurfaces of type $(A)$ in a complex space form ([3], [4], [5], [8] and [10] etc.). One of them, Cho and $\mathrm{Ki}$, one of the present authors, proved the following:

Theorem O-MR. ([4]). Let $\mathbf{M}$ be a connected real hypersurface of $\mathbf{M}_{n}(c)$ $c \neq 0$ whose shape operator $A$ commutes $R_{\xi}$, that is, $R_{\xi} A=A R_{\xi}$. Then $\mathbf{M}$ satisfies $\nabla_{\xi} R_{\xi}=0$ if and only if $\mathbf{M}$ is locally congruent to one of the following:

(1) In case that $\mathbf{M}_{n}(c)=\mathbf{P}_{n} \mathbb{C}$ with $\eta(A \xi) \neq 0$,

$\left(A_{1}\right)$ a geodesic hypersphere of radius $r$, where $0<r<\pi / 2$ and $r \neq$ $\pi / 4$

$\left(A_{2}\right)$ a tube of radius $r$ over a totally geodesic $\mathbf{P}_{k} \mathbb{C}(1 \leq k \leq n-2)$, where $0<r<\pi / 2$ and $r \neq \pi / 4$. 
(2) In case that $\mathbf{M}_{n}(c)=\mathbf{H}_{n} \mathbb{C}$,

$\left(A_{0}\right)$ a horosphere;

$\left(A_{1}\right)$ a geodesic hypersphere or a tube over a complex hyperbolic hyperplane $\mathbf{H}_{n-1} \mathbb{C}$;

$\left(A_{2}\right)$ a tube over a totally geodesic $\mathbf{H}_{k} \mathbb{C}(1 \leq k \leq n-2)$.

In this paper, we study a real hypersurface in a nonflat complex space form $\mathbf{M}_{n}(c)$ which satisfies $\nabla_{\xi} R_{\xi}=0$ and at the same time $R_{\xi} S=S R_{\xi}$, where $S$ denotes the Ricci tensor of the hypersurface. We give another characterization of real hypersurfaces of type $(A)$ in a nonflat complex space form by above two conditions. The main purpose of the present paper is to establish Theorem 6.1.

All manifolds in the present paper are assumed to be connected and of class $C^{\infty}$ and the real hypersurfaces are supposed to be orientable.

\section{Preliminaries}

Let $\mathbf{M}$ be a real hypersurface immersed in a complex space form $\mathbf{M}_{n}(c)$, and $N$ be a unit normal vector field of $\mathbf{M}$. By $\widetilde{\nabla}$ we denote the Levi-Civita connection with respect to the Fubini-Study metric tensor $\tilde{g}$ of $\mathbf{M}_{n}(c)$. Then the Gauss and Weingarten formulas are given respectively by

$$
\widetilde{\nabla}_{X} Y=\nabla_{X} Y+g(A X, Y) N, \quad \widetilde{\nabla}_{X} N=-A X
$$

for any vector fields $X$ and $Y$, where $g$ denoted the Riemannian metric tensor of $\mathbf{M}$ induced from $\tilde{g}$, and $A$ is the shape operator of $\mathbf{M}$ in $\mathbf{M}_{n}(c)$. For any vector field $X$ tangent to $M$ we put

$$
J X=\phi X+\eta(X) N, \quad J N=-\xi,
$$

where $J$ is the almost complex structure of $\mathbf{M}_{n}(c)$. Then we may see that $\mathbf{M}$ induces an almost contact metric structure $(\phi, \xi, \eta, g)$, that is

$$
\begin{gathered}
\phi^{2} X=-X+\eta(X) \xi, \quad g(\phi X, \phi Y)=g(X, Y)-\eta(X) \eta(Y), \\
\eta(\xi)=1, \quad \phi \xi=0, \quad \eta(X)=g(X, \xi)
\end{gathered}
$$

for any vector fields $X$ and $Y$ on $\mathbf{M}$.

Since $J$ is parallel, we verify from the Gauss and Weingarten formulas the following

$$
\begin{aligned}
\nabla_{X} \xi & =\phi A X \\
\left(\nabla_{X} \phi\right) Y & =\eta(Y) A X-g(A X, Y) \xi .
\end{aligned}
$$

Since the ambient manifold is of constant holomorphic sectional curvature $c$, we have the following Gauss and Codazzi equations respectively:

$$
\begin{aligned}
& R(X, Y) Z \\
= & \frac{c}{4}\{g(Y, Z) X-g(X, Z) Y+g(\phi Y, Z) \phi X-g(\phi X, Z) \phi Y \\
& -2 g(\phi X, Y) \phi Z\}+g(A Y, Z) A X-g(A X, Z) A Y,
\end{aligned}
$$




$$
\left(\nabla_{X} A\right) Y-\left(\nabla_{Y} A\right) X=\frac{c}{4}\{\eta(X) \phi Y-\eta(Y) \phi X-2 g(\phi X, Y) \xi\}
$$

for any vector fields $X, Y$ and $Z$ on $\mathbf{M}$, where $R$ denotes the Riemannian curvature tensor of $\mathbf{M}$.

Now let us denote by $\alpha=\eta(A \xi), \beta=\eta\left(A^{2} \xi\right), \gamma=\eta\left(A^{3} \xi\right), \mu^{2}=\beta-\alpha^{2}$, $h=\operatorname{trace} A$, and $\nabla f$ the gradient vector field of a function $f$ defined on $\mathbf{M}$. In the following, we use the same terminology and notation as above unless otherwise stated. We shall denote the Ricci tensor of type $(1,1)$ by $S$. Then it follows from (2.3) that

$$
S X=\frac{c}{4}\{(2 n+1) X-3 \eta(X) \xi\}+h A X-A^{2} X,
$$

which implies

$$
S \xi=\frac{c}{2}(n-1) \xi+h A \xi-A^{2} \xi .
$$

If we put $U=\nabla_{\xi} \xi$, then $U$ is orthogonal to the structure vector field $\xi$. Using (2.1) we see that

$$
\phi U=-A \xi+\alpha \xi,
$$

which shows that $g(U, U)=\beta-\alpha^{2}$. By definition of $U$ and (2.1) we verify that

$$
g\left(\nabla_{X} \xi, U\right)=g\left(A^{2} \xi, X\right)-\alpha g(A \xi, X) .
$$

Now, differentiating (2.7) covariantly along $M$ and using (2.1) and (2.2), we find that

$$
\begin{aligned}
& \eta(X) g(A U+\nabla \alpha, Y)+g\left(\phi X, \nabla_{Y} U\right) \\
=\quad & g\left(\left(\nabla_{Y} A\right) X, \xi\right)-g(A \phi A X, Y)+\alpha g(A \phi X, Y) .
\end{aligned}
$$

Together with (2.4) we obtain

$$
\left(\nabla_{\xi} A\right) \xi=2 A U+\nabla \alpha .
$$

From (2.9) we also have

$$
\nabla_{\xi} U=3 \phi A U+\alpha A \xi-\beta \xi+\phi \nabla \alpha,
$$

where we have used (2.1) and (2.8).

If $A \xi-\eta(A \xi) \xi \neq 0$, we can put

$$
A \xi=\alpha \xi+\mu W,
$$

where $W$ is a unit vector field orthogonal to $\xi$. Then from (2.7) it is clear that $U=\mu \phi W$ and hence $g(U, U)=\mu^{2}$, and $W$ is also orthogonal to $U$. Using (2.1) we see that

$$
\mu g\left(\nabla_{X} W, \xi\right)=g(A U, X) .
$$

From Gauss equation (2.3) we know that the structure Jacobi operator $R_{\xi}$ is given by

$$
R_{\xi} X=R(X, \xi) \xi=\frac{c}{4}\{X-\eta(X) \xi\}+\alpha A X-\eta(A X) A \xi
$$


for any vector field $X$ on $\mathrm{M}$.

In what follows we assume that $\mu \neq 0$ on $\mathbf{M}$, that is, $\xi$ is not a principal curvature vector field and we put $\Omega=\{p \in \mathbf{M} \mid \mu(p) \neq 0\}$. Suppose that $\Omega$ is not empty. Then $\Omega$ is an open subset of $\mathbf{M}$, and from now on we discuss our arguments on $\Omega$.

\section{3. $\xi$-parallel structure Jacobi operator}

Let $\mathbf{M}$ be a real hypersurface in a complex space form $\mathbf{M}_{n}(c), c \neq 0$ satisfying $\nabla_{\xi} R_{\xi}=0$, which means that the structure Jacobi operator is $\xi$-parallel.

Differentiating (2.14) covariantly, we find

$$
\begin{aligned}
& g\left(\left(\nabla_{X} R_{\xi}\right) Y, Z\right) \\
= & -\frac{c}{4}\left\{\eta(Z) g\left(\nabla_{X} \xi, Y\right)+\eta(Y) g\left(\nabla_{X} \xi, Z\right)+(X \alpha) g(A Y, Z)\right. \\
& +\alpha g\left(\left(\nabla_{X} A\right) Y, Z\right)-g(A \xi, Z)\left\{g\left(\left(\nabla_{X} A\right) \xi, Y\right)-g(A \phi A Y, X)\right\} \\
& -g(A \xi, Y)\left\{g\left(\left(\nabla_{X} A\right) \xi, Z\right)-g(A \phi A Z, X)\right\} .
\end{aligned}
$$

Together with (2.1) and (2.10) we have

$$
\begin{aligned}
& g\left(\left(\nabla_{\xi} R_{\xi}\right) Y, Z\right) \\
= & -\frac{c}{4}\{u(Y) \eta(Z)+u(Z) \eta(Y)\}+(\xi \alpha) g(A Y, Z) \\
& +\alpha g\left(\left(\nabla_{\xi} A\right) Y, Z\right)-g(A \xi, Z)\{3 g(A U, Y)+Y \alpha\} \\
& -g(A \xi, Y)\{3 g(A U, Z)+Z \alpha\},
\end{aligned}
$$

where $u$ is a 1 -form defined by $u(X)=g(U, X)$ for any vector field $X$.

From the last equation and $\nabla_{\xi} R_{\xi}=0$ we have

$$
\begin{aligned}
& \alpha\left(\nabla_{\xi} A\right) X+(\xi \alpha) A X \\
= & \frac{c}{4}\{u(X) \xi+\eta(X) U\}+\eta(A X)\{3 A U+\nabla \alpha\} \\
& +\{3 g(A U, X)+X \alpha\} A \xi .
\end{aligned}
$$

Putting $X=\xi$ in this and using (2.10) we get

$$
\alpha A U+\frac{c}{4} U=0
$$

which shows that $\alpha \neq 0$ on $\Omega$.

Putting $X=\alpha U$ in (3.1) and making use of (3.2) we find

$$
\alpha^{2}\left(\nabla_{\xi} A\right) U-\frac{c}{4}(\xi \alpha) U=\frac{c}{4} \alpha \mu^{2} \xi+\left\{\alpha(U \alpha)-\frac{3}{4} c \mu^{2}\right\} A \xi .
$$

Because of (3.2), the equation (2.11) turns out to be

$$
\alpha \nabla_{\xi} U=\frac{3}{4} c \mu W+\alpha^{2} A \xi-\alpha \beta \xi+\alpha \phi \nabla \alpha .
$$

Differentiating (3.2) covariantly along $\Omega$, we find

$$
(X \alpha) A U+\alpha\left(\nabla_{X} A\right) U+\alpha A\left(\nabla_{X} U\right)+\frac{c}{4} \nabla_{X} U=0 .
$$


If we replace $X=\alpha \xi$ in this equation and take account of (3.2) and (3.3), we can obtain

$$
\frac{c}{4} \alpha \mu^{2} \xi+\left\{\alpha(U \alpha)-\frac{3}{4} c \mu^{2}\right\} A \xi+\alpha^{2} A\left(\nabla_{\xi} U\right)+\frac{c}{4} \alpha \nabla_{\xi} U=0 .
$$

Together with (3.4) we get

(3.6) $\alpha A \phi \nabla \alpha+\frac{c}{4} \phi \nabla \alpha+(U \alpha) A \xi+\mu\left(\alpha^{2}+\frac{3}{4} c\right)\left\{A W-\mu \xi-\frac{1}{\alpha}\left(\mu^{2}-\frac{c}{4}\right) W\right\}=0$,

where we have used (2.12).

Using (2.4) and (2.7), we verify from (3.5) that

$$
\begin{aligned}
& \frac{c}{4}\{(Y \alpha) u(X)-(X \alpha) u(Y)\}+\frac{c}{4} \alpha^{2} \mu\{\eta(X) w(Y)-\eta(Y) w(X)\} \\
& +\alpha^{2}\left\{g\left(A \nabla_{X} U, Y\right)-g\left(A \nabla_{Y} U, X\right)\right\}+\frac{c}{4} \alpha \mathrm{d} u(X, Y)=0
\end{aligned}
$$

where $w$ is a 1 -form defined by $w(X)=g(W, X)$, and the exterior derivative $\mathrm{d} u$ of 1 -form $u$ is given by

$$
\mathrm{d} u(X, Y)=\frac{1}{2}\{Y u(X)-X u(Y)-u([X, Y])\} .
$$

If we replace $X$ by $U$ in (3.7), then we obtain

$$
\frac{c}{4}\left(\mu^{2} \nabla \alpha-(U \alpha) U\right)+\alpha^{2} A \nabla_{U} U+\frac{c}{4} \alpha \nabla_{U} U=0
$$

because $U$ and $W$ are mutually orthogonal.

Combining (2.9) to (3.1) and using the Codazzi equation (2.4) and (3.2), we obtain

$$
\begin{aligned}
\alpha^{2} \phi \nabla_{X} U= & -\alpha^{2}(X \alpha) \xi+\frac{c}{4} \alpha u(X) \xi-\alpha(\xi \alpha) A X-\frac{c}{4} \alpha^{2} \phi X \\
& +g(A \xi, X)\left(\alpha \nabla \alpha-\frac{3}{4} c U\right)+\left(\alpha(X \alpha)-\frac{3}{4} c u(X)\right) A \xi \\
& +\frac{c}{4}(u(X) \xi+\eta(X) U)-\alpha^{2} A \phi A X+\alpha^{3} \phi A X
\end{aligned}
$$

Applying this by $\phi$ and using (2.8) and (2.12), we have

$$
\begin{aligned}
& \alpha^{2} \nabla_{X} U+\alpha^{2} g(A W, X) \xi-\alpha g(A \xi, X) \phi \nabla \alpha \\
= & \alpha(\xi \alpha) \phi A X+\frac{c}{4} \alpha^{2}(X-\eta(X) \xi)+\frac{3}{4} c \mu g(A \xi, X) W+\alpha(X \alpha) U \\
& -\frac{3}{4} c u(X) U+\alpha^{3} A X-\frac{c}{4} \alpha \mu \eta(X) W-\alpha^{3} \eta(X) A \xi-\alpha^{2} \phi A \phi A X .
\end{aligned}
$$

Putting $X=U$ in (3.9) and taking account of (2.7), (2.12) and (3.2), we obtain

$$
\alpha^{2} \nabla_{U} U=-\frac{c}{4} \mu(\xi \alpha) W+\left\{\alpha(U \alpha)-\frac{3}{4} c \mu^{2}\right\} U+\frac{c}{4} \mu \alpha \phi A W
$$




\section{Real hypersurfaces satisfying $R_{\xi} S=S R_{\xi}$}

Let $\mathbf{M}$ be a real hypersurface in $\mathbf{M}_{n}(c), c \neq 0$ satisfying $R_{\xi} S=S R_{\xi}$, which means that the Ricci tensor $S$ of type $(1,1)$ and the Jacobi operator $R_{\xi}$ with respect to the structure vector field $\xi$ commute to each other. Then we have

$$
\begin{aligned}
& g\left(A^{3} \xi, Y\right) g(A \xi, X)-g\left(A^{3} \xi, X\right) g(A \xi, Y) \\
= & g\left(A^{2} \xi, Y\right) g\left(h A \xi-\frac{c}{4} \xi, X\right)-g\left(A^{2} \xi, X\right) g\left(h A \xi-\frac{c}{4} \xi, Y\right) \\
+ & \frac{c}{4} h\{g(A \xi, Y) \eta(X)-g(A \xi, X) \eta(Y)\},
\end{aligned}
$$

where we have used (2.6) and (2.14), which shows that

$$
\alpha A^{3} \xi=\left(\alpha h-\frac{c}{4}\right) A^{2} \xi+\left(\gamma-\beta h+\frac{c}{4} h\right) A \xi+\frac{c}{4} h(\beta-h \alpha) \xi .
$$

Combining above two equations, using (2.12) we see that

$$
\mu\left\{g\left(A^{2} \xi, Y\right) w(X)-g\left(A^{2} \xi, X\right) w(Y)\right\}=\beta\{\eta(Y) g(A \xi, X)-\eta(X) g(A \xi, Y)\} .
$$

Putting $Y=A \xi$ in this equation, we find that

$$
\mu^{2} g\left(A^{2} \xi, X\right)=\mu \gamma w(X)-\beta \alpha g(A \xi, X)+\beta^{2} \eta(X) .
$$

It follows that

$$
\mu^{2} A^{2} \xi=(\gamma-\beta \alpha) A \xi+\left(\beta^{2}-\alpha \gamma\right) \xi
$$

and consequently

$$
A^{2} \xi=\rho A \xi+(\beta-\rho \alpha) \xi
$$

where we have put $\mu^{2} \rho=\gamma-\beta \alpha$ and $\mu^{2}(\beta-\rho \alpha)=\beta^{2}-\alpha \gamma$ on $\Omega$.

From (2.12) and (4.2), we see that

$$
A W=\mu \xi+(\rho-\alpha) W
$$

and

$$
A^{2} W=\rho A W+(\beta-\rho \alpha) W
$$

because of $\mu \neq 0$.

Now, differentiating (4.3) covariantly, we find

$$
\left(\nabla_{X} A\right) W+A \nabla_{X} W=(X \mu) \xi+\mu \nabla_{X} \xi+X(\rho-\alpha) W+(\rho-\alpha) \nabla_{X} W
$$

which implies that

$$
\begin{aligned}
g\left(\left(\nabla_{X} A\right) W, W\right) & =-2 g(A U, X)+X \rho-X \alpha, \\
\mu\left(\nabla_{\xi} A\right) W & =(\rho-2 \alpha) A U-\frac{c}{4} U+\mu \nabla \mu,
\end{aligned}
$$

where we have used (2.4) and (2.13).

Replacing $X$ by $\xi$ in (4.5) and making use of (4.7), we find

$$
\begin{aligned}
& (\rho-2 \alpha) A U-\frac{c}{4} U+\mu \nabla \mu+\mu\left\{A \nabla_{\xi} W-(\rho-\alpha) \nabla_{\xi} W\right\} \\
= & \mu(\xi \mu) \xi+\mu^{2} U+\mu(\xi \rho-\xi \alpha) W .
\end{aligned}
$$


Since we have $\phi U=-\mu W$, it is verified that

$$
g(A U, X) \xi-\phi \nabla_{X} U=(X \mu) W+\mu \nabla_{X} W .
$$

Putting $X=\xi$ in this equation, and using (2.7) and (2.11), we obtain

$$
\mu \nabla_{\xi} W=3 A U-\alpha U+\nabla \alpha-(\xi \alpha) \xi-(\xi \mu) W,
$$

which shows that

$$
\xi \mu=W \alpha .
$$

Using the last two equations, (4.8) turns out to be

$$
\begin{aligned}
& 3 A^{2} U-2 \rho A U+A \nabla \alpha+\frac{1}{2} \nabla \beta-\rho \nabla \alpha+\left(\alpha \rho-\beta-\frac{c}{4}\right) U \\
= & 2 \mu(W \alpha) \xi+\mu(\xi \rho) W-(\rho-2 \alpha)(\xi \alpha) \xi .
\end{aligned}
$$

Differentiating (4.2) covariantly and making use of (2.1), we find

$$
\begin{aligned}
& \left(\nabla_{X} A\right) A \xi+A\left(\nabla_{X} A\right) \xi+A^{2} \phi A X-\rho A \phi A X \\
= & (X \rho) A \xi+\rho\left(\nabla_{X} A\right) \xi+X(\beta-\rho \alpha) \xi+(\beta-\rho \alpha) \phi A X,
\end{aligned}
$$

which together with (2.4) implies that

$$
\begin{aligned}
& \frac{c}{4}\{u(Y) \eta(X)-u(X) \eta(Y)\}+\frac{c}{2}(\rho-\alpha) g(\phi Y, X)-g\left(A^{2} \phi A X, Y\right) \\
& +g\left(A^{2} \phi A Y, X\right)+2 \rho g(\phi A X, A Y)-(\beta-\rho \alpha)\{g(\phi A Y, X)-g(\phi A X, Y)\} \\
= & g\left(A Y,\left(\nabla_{X} A\right) \xi\right)-g\left(A X,\left(\nabla_{Y} A\right) \xi\right)+(Y \rho) g(A \xi, X)-(X \rho) g(A \xi, Y) \\
& +Y(\beta-\rho \alpha) \eta(X)-X(\beta-\rho \alpha) \eta(Y) .
\end{aligned}
$$

If we replace $X$ by $\mu W$ to both sides of the last equation and take account of $(2.4),(2.10),(4.3),(4.4)$, and (4.7), then we obtain

$$
\begin{aligned}
& (3 \alpha-2 \rho) A^{2} U+2\left(\rho^{2}+\beta-2 \rho \alpha+\frac{c}{4}\right) A U+(\rho-\alpha)\left(\beta-\rho \alpha-\frac{c}{2}\right) U \\
= & \mu A \nabla \mu+(\alpha \rho-\beta) \nabla \alpha-\frac{1}{2}(\rho-\alpha) \nabla \beta+\mu^{2} \nabla \rho \\
& -\mu(W \rho) A \xi-\mu W(\beta-\rho \alpha) \xi .
\end{aligned}
$$

From (4.6) and (4.7) we see that

$$
W \mu=\xi \rho-\xi \alpha .
$$

5. Real hypersurfaces with $\nabla_{\xi} R_{\xi}=0$ and $R_{\xi} S=S R_{\xi}$

We will continue our arguments under the hypotheses $\nabla_{\xi} R_{\xi}=0$ and $R_{\xi} S=$ $S R_{\xi}$. Then (3.6) turns out to be

$$
\alpha A \phi \nabla \alpha+\frac{c}{4} \phi \nabla \alpha+(U \alpha) A \xi+\frac{1}{\alpha} \mu\left(\alpha^{2}+\frac{3}{4} c\right)\left(\rho \alpha+\frac{c}{4}-\beta\right) W=0
$$

by virtue of (4.3). Taking a inner product this with $W$ and using (4.3), we find

$$
\left(\beta-\rho \alpha-\frac{c}{4}\right)\left\{\alpha(U \alpha)-\mu^{2}\left(\alpha^{2}+\frac{3}{4} c\right)\right\}=0,
$$


which implies

$$
\alpha(U \alpha)=\mu^{2}\left(\alpha^{2}+\frac{3}{4} c\right)
$$

In fact, if not, then we have $\beta=\rho \alpha+\frac{c}{4}$ on this subset. So (4.2) becomes $A^{2} \xi=\rho A \xi+\frac{c}{4} \xi$. From this and (2.14) we see that $R_{\xi} A=A R_{\xi}$ on the set $\Omega$. According to Theorem $\operatorname{CK}([4])$, we verify that $\Omega=\emptyset$ because $\nabla_{\xi} R_{\xi}=0$ was assumed. Accordingly (5.1) is proved.

Because of (4.3) and (5.1), the equation (3.10) can be written as

$$
\alpha^{2} \nabla_{U} U=-\frac{c}{4} \mu(\xi \alpha) W+\alpha\left(\alpha \mu^{2}+\frac{c}{4}(\rho-\alpha)\right) U,
$$

which together with $(3.2)$ yields that

$$
\alpha^{2} A \nabla_{U} U=-\frac{c}{4} \mu(\xi \alpha) A W-\frac{c}{4}\left(\alpha \mu^{2}+\frac{c}{4}(\rho-\alpha)\right) U .
$$

Substituting (5.1) and the last two equations into (3.8) and making use of (4.3), we get

$$
\alpha \mu \nabla \alpha=\alpha \mu(\xi \alpha) \xi+\left(\rho \alpha-\alpha^{2}+\frac{c}{4}\right)(\xi \alpha) W+\left(\alpha^{2}+\frac{3}{4} c\right) \mu U,
$$

which enables us to obtain

$$
\mu \alpha(W \alpha)=\left(\rho \alpha-\alpha^{2}+\frac{c}{4}\right) \xi \alpha .
$$

From above two equations it follows that

$$
\alpha \nabla \alpha=\alpha(\xi \alpha) \xi+\alpha(W \alpha) W+\left(\alpha^{2}+\frac{3}{4} c\right) U .
$$

Using (3.2), the equation (3.1) can be written as

$$
\begin{aligned}
\alpha^{2}\left(\nabla_{\xi} A\right) X= & -\alpha(\xi \alpha) A X+\frac{c}{4} \alpha\{u(X) \xi+\eta(X) U\} \\
& +\left(\alpha(X \alpha)-\frac{3}{4} c u(X)\right) A \xi+\eta(A X)\left(\alpha \nabla \alpha-\frac{3}{4} c U\right) .
\end{aligned}
$$

Putting $X=\mu W$ in this and taking account of (3.2) and (4.7), we find

$$
\alpha\left(\frac{1}{2} \alpha \nabla \beta-\beta \nabla \alpha\right)+\frac{c}{4}\left(3 \beta-2 \alpha^{2}-\rho \alpha\right) U=-\mu \alpha(\xi \alpha) A W+\mu \alpha(W \alpha) A \xi,
$$

where we have used $\mu^{2}=\beta-\alpha^{2}$, or using (2.12), (4.3) and (5.3),

$$
\alpha^{2} \nabla \beta-\beta \nabla \alpha^{2}+\frac{c}{2}\left(3 \beta-2 \alpha^{2}-\rho \alpha\right) U=(\xi \alpha)\left\{2 \alpha(\rho \alpha-\beta) \xi+\frac{c}{2} A \xi\right\} .
$$


If we use (2.12), (4.3) and (5.4), then (3.9) is reduced to

$$
\begin{aligned}
& \alpha \nabla_{X} U+\alpha \mu^{2} \eta(X) \xi+g(A \xi, X)\left\{\mu \alpha W-\frac{1}{\mu}(W \alpha) U\right\} \\
= & (\xi \alpha) \phi A X+\frac{c}{4} \alpha(X-\eta(X) \xi)+\{(W \alpha) w(X)+\alpha u(X)\} U \\
& +\alpha^{2} A X-\alpha^{3} \eta(X) \xi-\frac{c}{4} \mu \eta(X) W-\alpha \phi A \phi A X,
\end{aligned}
$$

which implies that

$$
\begin{aligned}
\alpha \mathrm{d} u(X, Y)= & \mu\left(\rho \alpha-\alpha^{2}-\frac{c}{4}\right)(\eta(X) w(Y)-\eta(Y) w(X)) \\
& +\left\{\xi \alpha+\frac{\alpha}{\mu}(W \alpha)\right\}(\eta(X) u(Y)-\eta(Y) u(X)) \\
& +2(W \alpha)(w(X) u(Y)-w(Y) u(X)) \\
& +(\xi \alpha)\{g(\phi A X, Y)-g(\phi A Y, X)\} \\
& -\alpha\{g(\phi A \phi A X, Y)-g(\phi A \phi A Y, X)\} .
\end{aligned}
$$

Putting $X=\mu \xi$ in (5.6) and using (2.7), (3.2) and (4.3), we find

$$
\mu \mathrm{d} u(\xi, Y)=(W \alpha) u(Y)+\mu^{2}(\rho-\alpha) w(Y) .
$$

Since we have $\mu^{2}=\beta-\alpha^{2}$, it is, using (4.10) and (5.3), seen that

$$
\alpha(\xi \beta)=\left(2 \rho \alpha+\frac{c}{2}\right) \xi \alpha .
$$

On the other hand, we obtain from (3.1)

$$
\alpha^{2}(\xi h)=\alpha(2 \alpha-h) \xi \alpha+2 \mu \alpha(W \alpha)
$$

because of (2.12) and (3.2), which together with (5.3) gives

$$
\alpha^{2}(\xi h)=\left\{\alpha(2 \rho-h)+\frac{c}{2}\right\} \xi \alpha .
$$

By the way, combining (4.1) to (4.2), we verify that $(h-\rho)\left(\beta-\rho \alpha-\frac{c}{4}\right)=0$ on $\Omega$. Hence $h-\rho=0$ by virtue of $\beta-\rho \alpha-\frac{c}{4} \neq 0$ on $\Omega$. Accordingly (5.9) reformed as

$$
\alpha^{2}(\xi \rho)=\left(\rho \alpha+\frac{c}{2}\right) \xi \alpha .
$$

Using (5.9) and (5.10), we see that

$$
\xi\left(\rho \alpha-\alpha^{2}\right)=\xi \mu^{2}=\left\{2(\rho-\alpha)+\frac{c}{4 \alpha}\right\} \xi \alpha .
$$

Now, we put $\sigma=\beta-\rho \alpha$. Then we obtain

$$
\xi \sigma=0
$$

by virtue of (5.10) and (5.11).

Owing to (3.2) and (5.4), we verify from (4.9) that

$$
\nabla_{\xi} W=0 \text {. }
$$


If we take a inner product (5.5) with $U$ and use (5.1), then we get

$$
\frac{1}{2} \alpha(U \beta)=\left\{\beta \alpha+\frac{c}{4}(2 \alpha+\rho)\right\} \mu^{2} .
$$

This, together with (5.1) implies that

$$
\alpha \mu(U \mu)=\left\{\alpha \mu^{2}+\frac{c}{4}(\rho-\alpha)\right\} \mu^{2} .
$$

Applying (4.12) by $\alpha^{2} U$ and making use of (3.2), (5.1) and (5.14), we find

$$
\alpha^{2}(U \rho)=\alpha^{2}\left(2 \beta \rho-\rho^{2} \alpha-\beta \alpha\right)+\frac{c}{4} \alpha\left(2 \beta-\rho^{2}-\alpha^{2}\right)-\frac{c^{2}}{16} \rho
$$

which connected to (5.1) and (5.14) yields

$$
\begin{aligned}
(5.16) \alpha(U \sigma)= & \left\{2 \beta \alpha-\rho \alpha^{2}+\frac{c}{4}(4 \alpha-\rho)\right\} \mu^{2}+\alpha^{2}\left(\alpha \rho^{2}+\beta \alpha-2 \beta \rho\right) \\
& +\frac{c}{4} \alpha\left(\rho^{2}+\alpha^{2}-2 \beta\right)+\frac{c^{2}}{16} \rho .
\end{aligned}
$$

Now, we prove

Lemma 1. $\xi \alpha=0$ and $W \alpha=0$ on $\Omega$.

Proof. By the definition we have

$$
\alpha \mu \nabla \mu=\alpha\left(\frac{1}{2} \nabla \beta-\alpha \nabla \alpha\right) .
$$

Substituting (5.4) and (5.5) into this, and taking account of (2.12), (4.3) and (5.3), we obtain

$$
\frac{1}{2} \alpha^{2} \nabla \mu^{2}=\left\{\left(\alpha^{2}+\frac{c}{4}\right) \mu^{2}-\frac{c}{4} \sigma\right\} U+\varepsilon\left\{\left(\mu^{2}-\sigma\right) A \xi-\frac{c}{4} \xi\right\}
$$

where we have put $\varepsilon=\xi \alpha$. This is rewritten as

(5.17) $\frac{1}{2} a^{2}\left(Y \mu^{2}\right)=\left\{\left(\alpha^{2}+\frac{c}{4}\right) \mu^{2}-\frac{c}{4} \sigma\right\} u(Y)+\varepsilon\left\{\left(\mu^{2}-\sigma\right) \eta(A Y)-\frac{c}{4} \alpha \eta(Y)\right\}$.

Differentiating this with respect to a vector field $X$ again, and taking the skew-symmetric parts for $X$ and $Y$, we eventually have

$$
\begin{aligned}
& \left\{\alpha(X \alpha)+\varepsilon \eta(A X)+\left(\alpha^{2}+\frac{c}{4}\right) u(X)\right\}\left(Y \mu^{2}\right) \\
& -\left\{\alpha(Y \alpha)+\varepsilon \eta(A Y)+\left(\alpha^{2}+\frac{c}{4}\right) u(Y)\right\}\left(X \mu^{2}\right) \\
& +(X \alpha)\left(\frac{c}{4} \varepsilon \eta(Y)-2 \alpha \mu^{2} u(Y)\right)-(Y \alpha)\left(\frac{c}{4} \varepsilon \eta(X)-2 \alpha \mu^{2} u(X)\right)
\end{aligned}
$$




$$
\begin{aligned}
& +(X \sigma)\left(\varepsilon \eta(A Y)+\frac{c}{4} u(Y)\right)-(Y \sigma)\left(\varepsilon \eta(A X)+\frac{c}{4} u(X)\right) \\
= & \left\{\left(\alpha^{2}+\frac{c}{4}\right) \mu^{2}-\frac{c}{4} \sigma\right\} \mathrm{d} u(X, Y)+\alpha(\rho-\alpha) \varepsilon\left(2 g(A \phi A X, Y)-\frac{c}{2} g(\phi X, Y)\right) \\
& -\frac{c}{4} \alpha \varepsilon(g(\phi A X, Y)-g(\phi A Y, X))+\alpha(X \varepsilon)\left\{(\rho-\alpha) \eta(A Y)-\frac{c}{4} \eta(Y)\right\} \\
& -\alpha(Y \varepsilon)\left\{(\rho-\alpha) \eta(A X)-\frac{c}{4} \eta(X)\right\},
\end{aligned}
$$

where we have used the Codazzi equation (3.2). Putting $X=U$ and $Y=\alpha \xi$ in the last equation and making use of (3.2), (4.10) and (5.12), we find

$$
\begin{aligned}
& 4 \varepsilon \alpha^{2} \mu(U \mu)-2 \mu \alpha(W \alpha)\left\{\alpha(U \alpha)+\left(\alpha^{2}+\frac{c}{4}\right) \mu^{2}\right\} \\
& -2 \alpha^{2} \mu^{4} \varepsilon-\frac{c}{4} \varepsilon \alpha^{2}(U \sigma)-\varepsilon \alpha^{2}(U \sigma) \\
= & \left\{\left(\alpha^{2}+\frac{c}{4}\right) \mu^{2}-\frac{c}{4} \sigma\right\} \alpha \mathrm{d} u(\xi, U)-\alpha^{2}\left(\rho \alpha-\alpha^{2}-\frac{c}{4}\right) U \varepsilon \\
& +\frac{c}{2} \alpha(\alpha-\rho) \varepsilon \mu^{2}-\frac{c}{4} \varepsilon \alpha^{2} \mu^{2} .
\end{aligned}
$$

Let $\Omega_{0}$ be a set of points such that $(\xi \alpha)_{p} \neq 0$ at $p \in \Omega$ and suppose that $\Omega_{0} \neq \emptyset$. We then have from the last equation

$$
\begin{aligned}
\frac{1}{\varepsilon} \alpha^{2}\left(\rho \alpha-\alpha^{2}-\frac{c}{4}\right) U \varepsilon & =\alpha^{2}(U \sigma)-2 \alpha^{2} \mu^{4}+\frac{3}{2} c \alpha(\alpha-\rho) \mu^{2} \\
+ & \left(\rho \alpha-\alpha^{2}+\frac{c}{4}\right)\left\{5 \mu^{2}\left(\alpha^{2}+\frac{9}{4} c\right)-\frac{c}{4} \sigma\right\}
\end{aligned}
$$

on $\Omega_{0}$, where we have used $(5.1),(5.3),(5.7)$ and (5.15). Since we have $\rho \alpha-\alpha^{2}=$ $\mu^{2}-\sigma$, this is reformed as

$$
\begin{aligned}
& \frac{1}{\varepsilon} \alpha^{2}\left(\mu^{2}-\sigma-\frac{c}{4}\right) U \varepsilon-\alpha^{2}(U \sigma) \\
= & 3\left(\alpha^{2}+\frac{c}{4}\right) \mu^{4}+\left\{\frac{c}{4}\left(5 \sigma+\frac{3}{4} c\right)-\left(5 \alpha^{2}+\frac{9}{4} c\right)\left(\sigma-\frac{c}{4}\right)\right\} \mu^{2}+\frac{c}{4} \sigma\left(\sigma-\frac{c}{4}\right)
\end{aligned}
$$

on $\Omega_{0}$.

On the other hand, we have from (5.5)

$$
\alpha^{2}(Y \beta)-\beta\left(X \alpha^{2}\right)+\frac{c}{2}\left(2 \mu^{2}+\sigma\right) u(Y)=\varepsilon\left\{\frac{c}{2} \eta(A Y)-2 \alpha \sigma \eta(Y)\right\}
$$

Using the same method as that used to derive (5.18) from (5.17), we can deduce from the last equation the following :

$$
\begin{aligned}
& \alpha^{2}\left(\frac{c}{4}-\sigma\right) U \varepsilon-\alpha^{2} \varepsilon(U \sigma) \\
= & \frac{c}{4} \varepsilon \sigma\left(\rho \alpha-\alpha^{2}+\frac{c}{4}\right)+\left\{(4 \rho \alpha+c)\left(\alpha^{2}+\frac{3}{4} c\right)-4 \beta \alpha^{2}-c\left(\rho \alpha+2 \alpha^{2}\right)\right. \\
& \left.-\frac{c}{2}\left(\rho \alpha-\alpha^{2}+\frac{c}{4}\right)+\frac{3}{4} c \sigma-\frac{c^{2}}{8}\right\} \mu^{2}
\end{aligned}
$$


which shows that

$$
\begin{aligned}
& \frac{1}{\varepsilon} \alpha^{2}\left(\frac{c}{4}-\sigma\right)-\alpha^{2}(U \sigma) \\
= & \frac{3}{2} c \mu^{4}+\frac{c}{4} \sigma\left(\mu^{2}-\sigma+\frac{c}{4}\right)+\left(c \alpha^{2}-\frac{3}{4} c \sigma-4 \alpha^{2} \sigma+\frac{c^{2}}{2}\right) \mu^{2}
\end{aligned}
$$

on $\Omega_{0}$. From (5.19) and (5.20) it follows that

$$
\begin{aligned}
\frac{1}{\varepsilon}\left(\mu^{2}-\frac{c}{2}\right) \alpha^{2}(U \varepsilon)= & 3\left(\alpha^{2}-\frac{c}{4}\right) \mu^{4}+\frac{c}{2} \sigma\left(\sigma-\frac{c}{4}\right) \\
& +\left(\frac{c}{4} \alpha^{2}-\frac{c}{2} \sigma-\alpha^{2} \sigma+\frac{c^{2}}{4}\right) \mu^{2}
\end{aligned}
$$

on $\Omega_{0}$.

Now, we know from (5.4)

$$
Y \alpha=\varepsilon \eta(Y)+(W \alpha) w(Y)+\frac{1}{\alpha}\left(\alpha^{2}+\frac{3}{4} c\right) u(Y) .
$$

In the same way as above, it is, using (5.22), verified that

$$
\begin{aligned}
& \alpha^{2}\{(X \varepsilon) \eta(Y)-(Y \varepsilon) \eta(X)\}+\alpha^{2} \varepsilon\{g(\phi A X, Y)-g(\phi A Y, X)\} \\
& +\alpha^{2}\{X(W \alpha) w(Y)-Y(W \alpha) w(X)\} \\
& +\left(\alpha^{2}-\frac{3}{4} c\right)\{(X \alpha) u(Y)-(Y \alpha) u(X)\}+\alpha\left(\alpha^{2}+\frac{3}{4} c\right) \mathrm{d} u(X, Y)=0
\end{aligned}
$$

Putting $X=U$ and $Y=\xi$ in this and using (2.13), (3.2) and (5.13), we find

$$
\alpha^{2}(U \varepsilon)-\left(2 \alpha^{2}-\frac{3}{4} c\right) \mu^{2} \varepsilon-\frac{c}{4} \alpha \mu(W \alpha)+\alpha\left(\alpha^{2}+\frac{3}{4} c\right) \mathrm{d} u(\xi, U)=0
$$

which together with (5.3) and (5.7) implies that

$$
\frac{1}{\varepsilon} \alpha^{2}(U \varepsilon)=\left(\alpha^{2}-\frac{5}{4} c\right) \mu^{2}+\left(\sigma-\frac{c}{4}\right)\left(\alpha^{2}+\frac{c}{2}\right)
$$

on $\Omega_{0}$. Substituting this into (5.21), we find on $\Omega_{0}$

$$
\left(2 \alpha^{2}+\frac{c}{2}\right) \mu^{4}+\left(c \alpha^{2}-2 \sigma \alpha^{2}+c \sigma-\frac{c^{2}}{4}\right) \mu^{2}+\frac{c}{2}\left(\sigma-\frac{c}{4}\right)\left(\alpha^{2}+\sigma+\frac{c}{2}\right)=0 .
$$

Differentiating this with respect to a vector field $X$, and taking several choices of $X$, we eventually have $\varepsilon=0$ and hence $\Omega_{0}=\emptyset$, where we have used (5.1), (5.11), (5.12), (5.15) and (5.16). This means that $\xi \alpha=0$ is accomplished everywhere on $\Omega$. From this and (5.3) it follows that $W \alpha=0$. This completes the proof of Lemma 1.

According to Lemma 1, equations (5.4), (5.5) and (5.6) are reduced respectively to

$$
\begin{aligned}
& \alpha \nabla \alpha=\left(\alpha^{2}+\frac{3}{4} c\right) U, \\
& \alpha^{2} \nabla \beta-\beta \nabla \alpha^{2}+\frac{c}{2}\left(3 \beta-2 \alpha^{2}+\rho \alpha\right) U=0,
\end{aligned}
$$




$$
\begin{aligned}
\alpha \mathrm{d} u(X, Y)= & \mu\left(\rho \alpha-\alpha^{2}-\frac{c}{4}\right)(\eta(X) w(Y)-\eta(Y) w(X)) \\
& +\alpha\{g(\phi A \phi A Y, X)-g(\phi A \phi A X, Y)\}
\end{aligned}
$$

\section{Proof of main theorem}

We will continue our discussions for real hypersurfaces $\mathbf{M}$ in $\mathbf{M}_{n}(c), c \neq 0$ which satisfies $\nabla_{\xi} R_{\xi}=0$ and $R_{\xi} S=S R_{\xi}$ as in section 5 .

Using the formulas $(2.9),(3.2),(4.12),(5.2),(5.23),(5.24)$ and $(5.25)$, we will prove

Theorem 6.1. Let $\mathbf{M}$ be a real hypersurface with non-negative scalar curvature in a nonflat complex space form $\mathbf{M}_{n}(c)$ which satisfies $\nabla_{\xi} R_{\xi}=0$ and at the same time $R_{\xi} S=S R_{\xi}$, where $S$ denotes the Ricci tensor of $\mathbf{M}$. Then $\mathbf{M}$ is a Hopf hypersurface in $\mathbf{M}_{n}(c)$. Further, $\mathbf{M}$ is locally congruent to one of the following hypersurfaces:

(1) In case that $\mathbf{M}_{n}(c)=\mathbf{P}_{n} \mathbb{C}$ with $\eta(A \xi) \neq 0$,

$\left(A_{1}\right)$ a geodesic hypersphere of radius $r$, where $0<r<\pi / 2$ and $r \neq$ $\pi / 4$

$\left(A_{2}\right)$ a tube of radius $r$ over a totally geodesic $\mathbf{P}_{k} \mathbb{C}(1 \leq k \leq n-2)$, where $0<r<\pi / 2$ and $r \neq \pi / 4$.

(2) In case that $\mathbf{M}_{n}(c)=\mathbf{H}_{n} \mathbb{C}$,

$\left(A_{0}\right)$ a horosphere;

$\left(A_{1}\right)$ a geodesic hypersphere or a tube over a complex hyperbolic hyperplane $\mathbf{H}_{n-1} \mathbb{C}$

$\left(A_{2}\right)$ a tube over a totally geodesic $\mathbf{H}_{k} \mathbb{C}(1 \leq k \leq n-2)$.

Proof. Combining (5.23) to (5.24), we find

$$
\alpha \nabla \beta=\left\{2 \beta \alpha+\frac{c}{2}(2 \alpha+\rho)\right\} U
$$

Because of (2.12) and (3.2), we can write (4.12) as

$$
\begin{aligned}
& \frac{c}{4}(2 \rho-3 \alpha) A U+\left\{\alpha(\rho-\alpha)\left(\beta-\rho \alpha-\frac{c}{4}\right)-\frac{c}{2}\left(\rho^{2}+\beta-2 \rho \alpha+\frac{c}{4}\right)\right\} U \\
= & \alpha \mu A \nabla \mu+\alpha(\alpha \rho-\beta) \nabla \alpha-\frac{1}{2}(\rho-\alpha) \alpha \nabla \beta+\alpha \mu^{2} \nabla \rho-\mu^{2} \alpha(W \rho) W
\end{aligned}
$$

where we have used (4.13), (5.10) and Lemma 1.

Differentiating (5.23) covariantly and taking the skew-symmetric parts obtained, we find $\left(\alpha^{2}+\frac{3}{4} c\right) \mathrm{d} u(X, Y)=0$, where we have used (5.23) itself.

Similarly, from $(6.1)$ we have $\left\{2 \beta \alpha+\frac{c}{4}(2 \alpha+\rho)\right\} \mathrm{d} u(X, Y)=0$. Then we have by above two equations

$$
\alpha^{2}+\frac{3}{4} c=0, \quad \beta \alpha+\frac{c}{4}(2 \alpha+\rho)=0 .
$$


In fact, if not, then we have $\mathrm{d} u=0$. By taking a inner product (5.25) with $\xi$, we verify that $\rho=\alpha$. Hence (6.1) is led to $\frac{1}{2} \nabla \beta=\left(\beta+\frac{3}{4} c\right) U$. From this and (5.23) we see that $\mu \nabla \mu=\mu^{2} U$. Using these facts, (6.2) implies that $\left(\mu^{2}+\frac{c}{4}\right) A U=0$, which enables us to obtain $\mu^{2}+\frac{c}{4}=0$ and thus $\nabla \mu=0$. So we have $\mu=0$, a contradiction. Consequently we have $\nabla \alpha=0$ and $\nabla \beta=0$ by virtue of (6.1). Therefore we see that $\nabla \rho=0$ and hence $\nabla \mu=0$. Accordingly (6.2) is reformed as

$$
\frac{c}{4}(2 \rho-3 \alpha) A U+\left\{\alpha(\rho-\alpha)\left(\beta-\rho \alpha-\frac{c}{2}\right)-\frac{c}{2}\left(\rho^{2}+\beta-2 \rho \alpha+\frac{c}{4}\right)\right\} U=0,
$$

which together with (3.2) and (6.3) implies that

$$
\frac{c}{4}(3 \alpha-2 \rho)-2 \alpha\left(\rho^{2}+\beta-2 \rho \alpha+\frac{c}{4}\right)-3(\rho-\alpha)\left(\beta-\rho \alpha-\frac{c}{2}\right)=0 .
$$

From this and (6.3) we see that $6 \rho=7 \alpha$, which together with $(4.1),(4.2)$ and (6.3) implies that $\rho=h$.

On the other hand, we see, using (2.5), that the scalar curvature $r$ on $M$ is given by

$$
r=c\left(n^{2}-1\right)+h^{2}-T_{r}\left({ }^{t} A A\right),
$$

which together with $\alpha^{2}+\frac{3}{4} c=0$ and $6 h=7 \alpha$ will produce a contradiction because $r$ is non-negative. Accordingly we conclude that $\Omega=\emptyset$. Therefore we verify that the subset $\Omega$ in $\mathbf{M}$ on which $A \xi-\eta(A \xi) \xi \neq 0$ is an empty set. Namely, in $\mathbf{M}_{n}(c), c \neq 0$, very real hypersurface satisfying $\nabla_{\xi} R_{\xi}=0$ and $R_{\xi} S=S R_{\xi}$ is a Hopf hypersurface. Therefore we have $U=0$ and moreover, the function $\alpha$ should be constant on $\mathbf{M}([6])$. Thus, (3.1) is reformed as $\alpha \nabla_{\xi} A=0$. This, together with (2.4) and (2.9) yields $\alpha(A \phi-\phi A)=0$.

When the constant $\alpha$ identically vanishes, by Cecil and Ryan [2] we assert that $\mathbf{M}$ is a tube of radius $\frac{\pi}{4}$ over certain Kähler submanifold in $\mathbf{P}_{n} \mathbb{C}$. But we here note that any Hopf hypersurfaces in $\mathbf{H}_{n} \mathbb{C}$ the function $\alpha$ never vanishing (see [1], [8], [9]). For the non-vanishing constant $\alpha$, by virtue of Theorem OMR due to Okumura [10] for $c>0$ and Montiel and Romero [8] for $c<0$ respectively we complete the proof of Theorem 6.1 .

From above arguments and (6.3) we have

Theorem 6.2. Let $\mathbf{M}$ be a real hypersurface in a complex projective space $P_{n}(\mathbb{C})$ which satisfies $\nabla_{\xi} R_{\xi}=0$ and at the same time $R_{\xi} S=S R_{\xi}$, where $S$ denotes the Ricci tensor of $\mathbf{M}$. Then $\mathbf{M}$ is a Hopf hypersurface in $P_{n}(\mathbb{C})$. Further, $\mathbf{M}$ is locally congruent to one of the following hypersurfaces:

$\left(A_{1}\right)$ a geodesic hypersphere of radius $r$, where $0<r<\pi / 2$ and $r \neq \pi / 4$;

$\left(A_{2}\right)$ a tube of radius $r$ over a totally geodesic $\mathbf{P}_{k} \mathbb{C}(1 \leq k \leq n-2)$, where $0<r<\pi / 2$ and $r \neq \pi / 4$

where $\eta(A \xi) \neq 0$. 
Acknowledgements. The first author would like to thank the faculty for their warm hospitality when he visited Northeastern University in June 2006.

\section{References}

[1] J. Berndt, Real hypersurfaces with constant principal curvatures in complex hyperbolic space, J. Reine Angew. Math. 395 (1989), 132-141.

[2] T. E. Cecil and P. J. Ryan, Focal sets and real hypersurfaces in complex projective space, Trans. Amer. Math. Soc. 269 (1982), no. 2, 481-499.

[3] J. T. Cho and U-H Ki, Real hypersurfaces of a complex projective space in terms of the Jacobi operators, Acta Math. Hungar. 80 (1998), no. 1-2, 155-167.

[4] _ Real hypersurfaces in a complex space form with the symmetric Reeb flow, preprint.

[5] U-H. Ki, J. D. Pérez, F. G. Santos, and Y. J. Suh, Real hypersurfaces in complex space forms with $\xi$-parallel Ricci tensor and structure Jacobi operator, J. Korean Math. Soc. 44 (2007), no. 2, 307-326.

[6] U-H. Ki and Y. J. Suh, On real hypersurfaces of a complex space form, Math. J. Okayama Univ. 32 (1990), 207-221.

[7] M. Kimura, Real hypersurfaces and complex submanifolds in complex projective space, Trans. Amer. Math. Soc. 296 (1986), no. 1, 137-149.

[8] S. Montiel and A. Romero, On some real hypersurfaces of a complex hyperbolic space, Geometriae Dedicata 20 (1986), no. 2, 245-261.

[9] R. Niebergall and P. J. Ryan, Real hypersurfaces in complex space forms. (English summary) Tight and taut submanifolds (Berkeley, CA, 1994), 233-305, Math. Sci. Res. Inst. Publ., 32, Cambridge Univ. Press, Cambridge, 1997.

[10] M. Okumura, On some real hypersurfaces of a complex projective space, Trans. Amer. Math. Soc. 212 (1975), 355-364.

[11] M. Ortega, J. D. Pérez, and F. G. Santos, Non-existence of real hypersurfaces with parallel structure Jacobi operator in nonflat complex space forms, to appear in Rocky Mountain J. Math.

[12] J. D. Pérez, F. G. Santos, and Y. J. Suh, Real hypersurfaces in complex projective space whose structure Jacobi operator is Lie $\xi$-parallel, Diff. Geom. and its Appl. 22 (2005), no. $2,181-188$.

[13] R. Takagi, On homogeneous real hypersurfaces in a complex projective space, Osaka J. Math. 10 (1973), 495-506.

\section{U-HANG KI}

The National ACADEMy of Sciences

SEOUL 137-044, KOREA

E-mail address: uhangki20050yahoo.co.kr

HUILI LIU

DEPARTMENT OF MATHEMATICS

NORTHEASTERN UNIVERSITY

Shenyang 110004, P. R. China

E-mail address: liuhlomail.neu.edu.cn 